\title{
Serum uric acid levels and hyperuricemia in patients with psoriasis: a hospital-based cross-sectional study*
}

\author{
Xin-Yu Gui ${ }^{1}$, Hong-Zhong Jin ${ }^{1}$, Zhen-Jie Wang ${ }^{2}$, Teng-Da Xu ${ }^{2}$
}

DOI: http:/ /dx.doi.org/10.1590/abd1806-4841.20187547

\begin{abstract}
A hospital-based cross-sectional study was performed, including 117 psoriatic patients and 117 controls matched for age, gender, and body mass index. Psoriatic patients had higher levels of serum uric acid (6.25 $\pm 1.62 \mathrm{vs} 5.71 \pm 1.35 \mathrm{mg} / \mathrm{dl}$; $\mathrm{P}=0.019)$ and significantly greater prevalence of hyperuricemia $(31.6 \% \mathrm{vs} 16.2 \% ; \mathrm{P}=0.009)$ than individuals without psoriasis. Psoriatic patients had significantly higher serum uric acid than controls in subjects without metabolic syndrome. Multivariate logistic regression analysis showed that psoriasis can be a strong predictor of hyperuricemia (odds ratio 2.61 ; $95 \%$ confidence interval 1.34-5.00; $\mathrm{P}=0.004)$.
\end{abstract}

Keywords: Body mass index; Metabolic syndrome X; Psoriasis; Uric acid

Psoriasis is a chronic inflammatory and immune-mediated disease that affects the skin and involves several other organs and systems. ${ }^{1}$ Recent studies have linked psoriasis to metabolic syndrome. ${ }^{2,3}$ Similarly, it is generally accepted that hyperuricemia frequently accompanies psoriasis and psoriatic arthritis. ${ }^{4}$ The present study investigated the correlation between hyperuricemia and psoriasis by comparing serum uric acid (SUA) levels and prevalence of hyperuricemia in psoriatic patients and controls.

This descriptive, cross-sectional study included 117 adult psoriatic patients from the Department of Dermatology at Peking Union Medical College Hospital. Exclusion criteria were systemic antipsoriatic treatment within 3 months of enrolment and use of medications known to affect SUA level. Disease severity was scored using the Psoriasis Area and Severity Index (PASI). The control group consisted of 117 adult individuals selected in a 1:1 ratio matched for age, sex, and body mass index (BMI) with psoriatic patients from the Department of Physical Examination Centre. ${ }^{5}$ Metabolic syndrome was defined according to the criteria of the Chinese Diabetes Society (CDS) in 2004, which was more suitable for Chinese individuals (Table 1). ${ }^{6}$ Hyperuricemia was defined as SUA level $\geq 7 \mathrm{mg} / \mathrm{dl}$ in men and $\geq 6 \mathrm{mg} / \mathrm{dl}$ in women, or allopurinol use. Statistical analysis was performed using SPSS 20 (IBM, Armonk, NY, USA) and GraphPad Prism software packages.

Patients with psoriasis had a higher prevalence of dyslipidemia ( $57.3 \%$ vs $39.3 \%$, p=0.006), whereas high blood pressure, hyperglycemia, and metabolic syndrome did not differ significantly

Received 17 August 2017.

Accepted 13 December 2017.

* Work conducted at the Peking Union Medical College Hospital, Beijing, China.

Financial support: The present study was supported by the Medical and Health Science and Technology Innovation Project of the Chinese Academy of Medical Sciences (NO.2017-12M-3-020).

Conflict of interest: None.

Department of Dermatology, Peking Union Medical College Hospital, Beijing, China.

Department of Physical Examination Center, Peking Union Medical College Hospital, Beijing, China.

MAILING AdDRESS:

Hong-Zhong Jin

E-mail: jinhongzhong@263.net

(C)2018 by Anais Brasileiros de Dermatologia

(cc) BY-NC 
TABLE 1: Diagnostic criteria for metabolic syndrome recommended by the Chinese Diabetes Society ( $\geq 3$ of the following criteria are required)

\begin{tabular}{ll} 
Components & Index \\
\hline $\begin{array}{l}\text { Overweight or obesity } \\
\text { Hyperglycemia }\end{array}$ & $\mathrm{BMI} \geq 25.0 \mathrm{~kg} / \mathrm{m} 2$ \\
High blood pressure & FBG $\geq 6.1 \mathrm{mmol} / \mathrm{L}$ and/or receiving treatment for diabetes mellitus \\
Dyslipidemia & $\begin{array}{l}\text { Fasting blood triglycerides } \geq 1.7 \mathrm{mmol} / \mathrm{L} \text { and } / \text { or fasting blood } \mathrm{HDL} \text { cholesterol }<0.9 \mathrm{mmol} / \mathrm{L} \text { for men } \\
\text { or }<1.0 \mathrm{mmol} / \mathrm{L} \text { for women }\end{array}$ \\
\hline
\end{tabular}

FBG: fasting blood glucose; HDL: high-density lipoprotein

Source: Advice, $2004{ }^{6}$

TABLE 2: Predictors of hyperuricemia in the total sample of participants: multivariate logistic regression models

\begin{tabular}{lllll} 
Clinical characteristics & Multivariate model 1 & P value & Multivariate model 2 & P value \\
\hline Age, y & $0.986(0.965-1.007)$ & 0.179 & $0.981(0.956-1.006)$ & 0.140 \\
Sex, F vs M & $0.520(0.222-1.217)$ & 0.132 & $0.810(0.312-2.100)$ & 0.665 \\
Psoriasis, & $2.606(1.359-4.996)$ & 0.004 & $2.387(1.136-5.014)$ & 0.022 \\
Metabolic syndrome & $2.997(1.400-6.419)$ & 0.005 & - & - \\
BMI, kg/m2 & - & - & $1.205(1.087-1.336)$ & $<0.001$ \\
Hyperglycemia & - & - & $0.726(0.248-2.123)$ & 0.559 \\
High blood pressure & - & - & $1.857(0.842-4.094)$ & 0.125 \\
Dyslipidemia & - & - & $3.582(1.588-8.079)$ & 0.002 \\
\hline
\end{tabular}

Model 1 = age, sex, presence of psoriasis, and metabolic syndrome;

Model 2 = age, sex, presence of psoriasis, BMI, hyperglycemia, high blood pressure, and dyslipidemia.

between the two groups. SUA levels were significantly higher in psoriatic patients compared to controls $(6.25 \pm 1.62$ vs $5.71 \pm 1.35 \mathrm{mg} /$ $\mathrm{dl} ; \mathrm{p}=0.019)$, and hyperuricemia was significantly more common in psoriatic patients than in control subjects $(31.6 \%$ vs $16.2 \%$; $=0.009)$. Male and female psoriatic patients had significantly higher SUA levels than their control counterparts $(6.61 \pm 1.6$ vs $6.06 \pm 1.2 \mathrm{mg} / \mathrm{dl}$ in men and $5.12 \pm 1.1 \mathrm{vs} 4.58 \pm 1.0 \mathrm{mg} / \mathrm{dl}$ in women). However, the results changed when participants were further stratified according to presence of metabolic syndrome. In subjects with metabolic syndrome, SUA levels were similar between psoriatic patients and controls ( $p=0.3537$ ). Meanwhile, SUA levels were significantly higher in psoriatic patients than controls in subjects without metabolic syndrome $(p=0.0035)$. Multivariate logistic regression showed that both psoriasis and metabolic syndrome, independently of each other, were risk factors for hyperuricemia (Table 2). Additionally, SUA level was positively correlated with BMI $(r=0.46$; $p<0.001)$, serum triglycerides $(r=0.30 ; p<0.001)$, and $\operatorname{sex}(r=0.42 ; p<0.001)$ but was not significantly correlated with age or the duration of psoriasis. Among psoriatic patients, mean SUA was higher in patients with PASI $\geq 10(n=112)$ than in those with PASI $<10(n=5)$ (SUA: $5.5 \pm 1.1$ vs $6.3 \pm 1.6 \mathrm{mg} / \mathrm{dl}$ ). However, SUA was not significantly associated with PASI.

In this study, the prevalence of asymptomatic hyperuricemia was approximately twice as high in psoriatic patients than in matched controls. Moreover, psoriasis was a significantly positive predictor of hyperuricemia after adjusting for associated baseline characteristics such as age, sex, BMI, and other features of metabolic syndrome. To date, convincing evidence has indicated that psoriasis is independently associated with hyperuricemia. ${ }^{5,7-9}$ In line with the previous studies, our findings also demonstrated that psoriasis itself might contribute directly to hyperuricemia. However, our study did not find a correlation between serum uric acid levels and severity of psoriasis.

Our findings can have clinical implications, since elevated SUA independently predicts the development of cardiovascular disease events and mortality in nonpsoriatic populations. ${ }^{5}$ Some studies have suggested that psoriatic patients with hyperuricemia show remarkable improvement in their psoriasis when treated for hyperuricemia. ${ }^{7}$ Wei et al. ${ }^{10}$ concluded that higher doses of allopurinol contributed to lower risks of both cardiovascular events and mortality. We recommend that SUA should be routinely measured in patients with psoriasis, especially in those with obesity, hypertension, or dyslipidemia; drug treatment of hyperuricemia might be a useful strategy for psoriasis.

The limitation of our study was that the patients enrolled were biased toward having higher disease severity, since the study population was based in a tertiary medical center. Large interventional clinical trials are needed to confirm the association between hyperuricemia and psoriasis and the efficacy of high-dose allopurinol treatment in psoriatic patients with hyperuricemia. $\square$ 


\section{REFERENCES}

1. Grozdev I, Korman N, Tsankov N. Psoriasis as a systemic disease. Clin Dermatol. 2014;32:343-50

2. Azfar RS, Gelfand JM. Psoriasis and metabolic disease: epidemiology and pathophysiology. Curr Opin Rheumatol. 2008;20:416-22.

3. Machado-Pinto J, Diniz Mdos S, Bavoso NC. Psoriasis: new comorbidities. An Bras Dermatol. 2016;91:8-14.

4. Lambert JR, Wright V. Serum uric acid levels in psoriatic arthritis. Ann Rheum Dis. 1977;36:264-67.

5. Gisondi P, Targher G, Cagalli A, Girolomoni G. Hyperuricemia in patients with chronic plaque psoriasis. J Am Acad Dermatol. 2014;70:127-30.
6. Advice on Metabolic Syndrome from Chinese Diabetes Society. Chin J Diabetes. 2004;12:156-61.

7. Goldman M. Uric acid in the etiology of psoriasis. Am J Dermatopathol. 1981 Winter;3:397-404

8. Zhang Q, Zhang C, Song X, Lin H, Zhang D, Meng W, et al. A longitudinal cohortbased association study between uric acid level and metabolic syndrome in Chinese Han urban male population. BMC Public Health. 2012;12:419.

9. Lai YC, Yew YW. Psoriasis and uric acid: a population-based cross-sectional study. Clin Exp Dermatol. 2016;41:260-6.

10. Wei L, Mackenzie IS, Chen Y, Struthers AD, MacDonald TM. Impact of allopurino use on urate concentration and cardiovascular outcome. Br J Clin Pharmacol. 2011;71:600-7.

\section{AUTHORS'CONTRIBUTIONS}

Xin-Yu Gui (iD) ORCID 0000-0002-0589-8732

Statistical analysis, Approval of the final version of the manuscript, Preparation and writing of the manuscript, Collecting, analysis and interpretation of data, Effective participation in research orientation, Critical review of the literature

Hong-Zhong Jin (iD) ORCID 0000-0001-6234-1554

Approval of the final version of the manuscript, Design and planning of the study, Effective participation in research orientation, Intellectual participation in propaedeutic and/ or therapeutic conduct of studied cases, Critical review of the manuscript
Zhen-Jie Wang

iD ORCID 0000-0002-6752-1690

Statistical analysis, Approval of the final version of the manuscript, Design and planning of the study, Collecting, analysis and interpretation of data, Intellectual participation in propaedeutic and/or therapeutic conduct of studied cases

$\begin{array}{ll}\mathrm{T} e n g-\mathrm{Da} \mathrm{Xu} & \text { (iD) ORCID 0000-0003-1774-3786 }\end{array}$

Approval of the final version of the manuscript, Design and planning of the study, Collecting, analysis and interpretation of data, Effective participation in research orientation, Intellectual participation in propaedeutic and/or therapeutic conduct of studied cases

How to cite this article: Gui XY, Jin HZ, Wang ZJ, Xu TD. Serum uric acid levels and hyperuricemia in patients with psoriasis: a hospital-based cross-sectional study. An Bras Dermatol. 2018;93(5):761-3. 